\title{
PRIMER REGISTRO DE LA DIATOMEA EPIBIONTE PSEUDOHIMANTIDIUM PACIFICUM Y DE OTRAS ASOCIACIONES SIMBIÓTICAS EN EL GOLFO DE CALIFORNIA
}

\author{
Ismael Gárate-LizÁrraga ${ }^{1}$ y María del Socorro Muñetón-Gómez ${ }^{1,2}$
}

\begin{abstract}
${ }^{1}$ Instituto Politécnico Nacional, Centro Interdisciplinario de Ciencias Marinas, Departamento de Plancton y Ecología Marina, Avenida Instituto Politécnico Nacional s/n, Colonia Playa Palo de Santa Rita, Apdo. postal 592, 23096 La Paz, Baja California Sur, México. igarate@ipn.mx; igarateipn@yahoo.com.mx ${ }^{2}$ Centro de Estudios Tecnológicos del Mar núm. 4, Avenida Instituto Politécnico Nacional y Calle Cetmar s/n, Colonia Playa Palo de Santa Rita, Apdo. postal 585, 23096 La Paz, Baja California Sur, México. mmunetong@ipn.mx
\end{abstract}

\section{RESUMEN}

En México existe poca información sobre las asociaciones simbióticas entre diatomeas y ciliados, así como con otras especies de microalgas y particularmente con crustáceos planctónicos marinos. Es por ello que en este trabajo se presenta el primer registro de la diatomea Pseudohimantidium pacificum en epibiosis con el copépodo Farranula gibbula y otras asociaciones entre microalgas y ciliados marinos en la Bahía de La Paz. Las muestras biológicas se recolectaron mediante una red de plancton con una luz de malla de $20 \mu \mathrm{m}$, en diversas áreas de la Bahía de La Paz. Se encontraron 62 copépodos machos de F. gibbula, de los cuales 27 presentaban sobre su caparazón un ramillete compuesto por la diatomea epibionte $P$. pacificum. Los valores promedio de abundancia de $P$. pacificum por estación de muestreo variaron entre 16 y 82 ejemplares/ copépodo. Otras diatomeas penales fueron observadas como epibiontes de copépodos del género Acartia. Asimismo se encontraron asociaciones simbióticas adicionales entre ciliados, microalgas y cianobacterias, destacando las siguientes: Chaetoceros coarctatumVorticella oceanica, Leptocylindrus mediterraneus-Solenicola setigera. Se registra por primera vez la agrupación entre la diatomea Fragilariopsis doliolus y el silicoflagelado Dictyocha pentagona con un ciliado de tipo Vorticella.

Palabras clave: copépodos, epibionte, Farranula gibbula, Golfo de California, microalgas, Pseudohimantidium pacificum. 


\begin{abstract}
In Mexico there is little information about the symbiotic associations among diatoms on other marine microalgae and particularly, ciliates and marine planktonic crustaceans. For that reason, we report the first record of the epizoic symbiosis between the copepod Farranula gibbula and the diatom Pseudohimantidium pacificum as well as other microalgae associations with marine ciliates in Bahía de La Paz. Biological samples were collected using a $20-\mu \mathrm{m}$ mesh plankton net in six sampling sites in Bahía de La Paz. Sixty-two male copepods of F. gibbula were found. From these, 27 specimens showed a bouquet composed of the diatom $P$. pacificum on its carapace. Average abundance values of P. pacificum for each sampling station ranged from 16 to 82 diatoms per copepod. Pennate diatoms were observed as epibionts on copepods belonging to the genus Acartia. In addition, other symbiotic relationships among ciliates, microalgae and cianobacteria were also encountered, outstanding the following ones: Chaetoceros coarctatum-Vorticella oceanica, Leptocylindrus mediterraneus-Solenicola setigera. The association between the diatom Fragilariopsis doliolus and the silicoflagellate Dictyocha pentagona with a Vorticella-like ciliate is reported for the first time.
\end{abstract}

Keywords: copepods, epibiont, Farranula gibbula, Gulf of California, microalgae, Pseudohimantidium pacificum.

\title{
INTRODUCCIÓN
}

La epibiosis es un tipo de simbiosis en la que un organismo vive sobre la capa superficial del otro. A estos organismos no parásitos, que viven por lo menos una fase de su ciclo vital encima de otro de mayor tamaño, al cual generalmente no le causa ningún problema, se les llama epibiontes (Lincoln et al., 1995). Los caparazones de algunos crustáceos marinos a menudo sirven de sustrato a diversas especies de algas, virus, u hongos (Green, 1974; Ho y Perkins, 1985). La presencia de algunas diatomeas y ciliados como epibiontes sobre los exoesqueletos de organismos zooplanctónicos es un fenómeno común en los copépodos marinos (Hiromi et al., 1985; Carman y Dobbs, 1997; Ohtsuka et al., 2004). Aunque esta relación ha sido documentada desde el siglo IX, se ha puesto poca atención a tal tipo de interacciones (Hiromi et al., 1985).

Entre las principales especies de diatomeas epibiontes sobre copépodos marinos se encuentran Pseudohimantidium pacificum Hustedt \& Krasske, Falcula hya- 
lina Takano, Sceptronema orientale Takano y Licmophora unidenticulata Takano (Gibson, 1978; Takano, 1983; González y Vergara, 1984; Hiromi et al., 1985). P. pacificum fue descrita por primera vez para las costas chilenas $\left(20^{\circ} 39^{\prime} \mathrm{S}, 70^{\circ} 20^{\prime} \mathrm{W}\right.$ ) (Krasske, 1941) y posteriormente fue registrada para otras regiones del mundo (Simonsen, 1970; 1974). Sin embargo, para México no existen registros de esta especie, ya que los diferentes estudios que se han realizado sobre diatomeas epibiontes han sido con referencia a pastos y macroalgas marinas (Siqueiros-Beltrones et al., 1985, 2002; Siqueiros-Beltrones y Argumedo-Hernández 2005).

Por otra parte, las asociaciones simbióticas entre diatomeas y dinoflagelados con otros organismos del plancton son comunes en aguas del Golfo de California, siendo en su mayoría casos de epifitismo y en menor proporción de endosimbiosis (Cupp, 1943; Gárate-Lizárraga et al., 1990, 2008; Gárate-Lizárraga y Siqueiros-Beltrones, 1998; Alonso-Rodríguez et al., 2003; Gárate-Lizárraga y Martínez-López, 2004) y parasitismo (Gárate-Lizárraga y Siqueiros-Beltrones, 2003; Gárate-Lizárraga et al., 2006; Coats et al., 2008).

Este estudio es una contribución parcial de los resultados obtenidos del monitoreo que el grupo de Fitoplancton del Centro Interdisciplinario de Ciencias Marinas (CICIMAR) realizó mensualmente en la Bahía de La Paz, en el Golfo de California, durante el periodo comprendido entre 2006 y 2008 en tres estaciones de muestreo fijas, y cuyo objetivo es dar a conocer el primer caso de epizoismo de la diatomea P. pacificum sobre el copépodo Farranula gibbula, de otras diatomeas sobre copépodos, así como de algunas relaciones simbióticas adicionales encontradas en las aguas de la bahía.

\section{ÁREA DE ESTUDIO}

La Bahía de La Paz está ubicada sobre el margen suroriental de la Península de Baja California entre los $24^{\circ} 10^{\prime}-24^{\circ} 47^{\prime} \mathrm{N}$ y los $110^{\circ} 20^{\prime}$ - $110^{\circ} 44^{\prime} \mathrm{W}$ (Fig. 1), con un área aproximada de $2635 \mathrm{~km}^{2}$ (Cruz-Orozco et al., 1989). El promedio anual de la temperatura del agua en la Bahía de La Paz, es de $24.7^{\circ} \mathrm{C}$ con un intervalo de 20 a $29.5^{\circ} \mathrm{C}$. La salinidad media es de 36 ppm (Villaseñor-Casales, 1979) en función del hecho de que no existen aportes de agua dulce en el área y la evaporación se intensifica en el período cálido. La bahía está protegida por la Isla San José al norte y al este por el archipiélago de las Islas Espíritu Santo (De Silva-Dávila y PalomaresGarcía, 2002). Se comunica con el Golfo de California por medio de dos bocas, una al noreste (Boca grande) y otra al este (Boca chica o Canal de San Lorenzo). 


\section{MÉTODOS}

Las muestras utilizadas para este estudio en particular fueron recolectadas del 13 al 16 de diciembre del 2006 en seis estaciones (Fig. 1). En los meses de enero de 2007 y febrero, septiembre y octubre de 2008 sólo se hicieron en los sitios 1, 2 y 3. En cada uno de ellos se realizaron tres arrastres superficiales mediante una red de fitoplancton de $20 \mu \mathrm{m}$ y se midió la temperatura del agua con un termómetro de cubeta (Kahlsico).

De las tres muestras obtenidas en los arrastres, dos se fijaron con lugol y formol, respectivamente y en la tercera alícuota no se utilizó fijador. Posteriormente

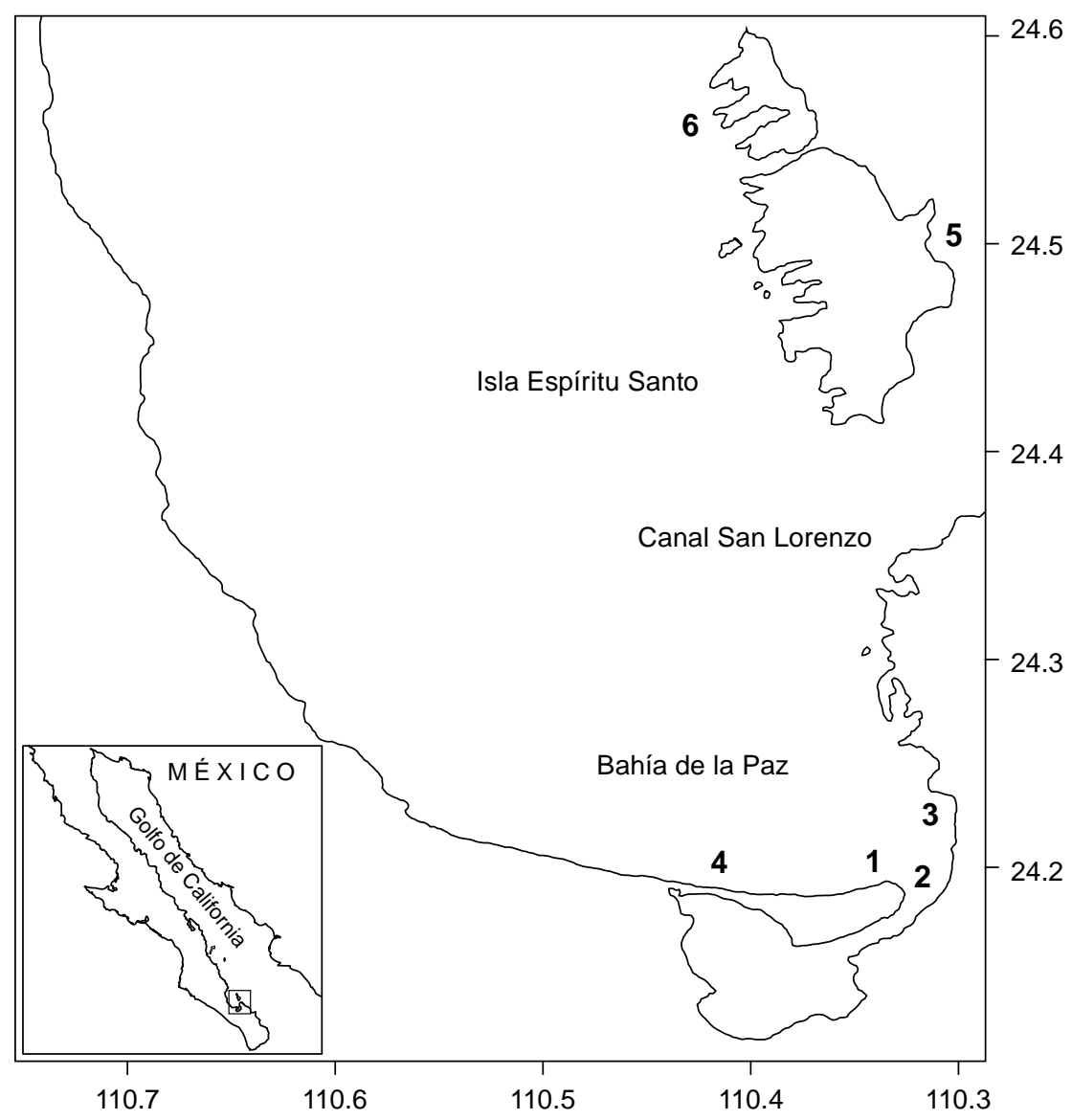

Fig. 1. Área de estudio y localización de las estaciones de muestreo (1-6). 
se revisaron minuciosamente mediante un microscopio invertido Zeiss, utilizando cámaras de sedimentación (Utermöhl, 1958), con el fin de encontrar la presencia de copépodos infestados con diatomeas y contar el número de estas últimas.

Las diatomeas se identificaron utilizando los trabajos de Gibson (1979), Hiromi et al. (1985) y Skovgaard y Saiz (2006). Las características morfológicas propias de Pseudohimantidium pacificum han sido descritas y resumidas en los trabajos de Gibson (1979) y Rivera et al. (1986). Para la identificación del copépodo se usó el catálogo de de Palomares-García et al. (1998).

Las imágenes que aparecen en la figura 2 se tomaron en un microscopio invertido Zeiss utilizando una cámara fotográfica digital Panasonic, y las de la figura 4 se obtuvieron con un microscopio Olympus CK2 (Japón), mediante una cámara digital integrada CCD-Pro-series y el procesador de imágenes Image-Pro-express.

\section{RESULTADOS Y DISCUSIÓN}

Epibiosis de Pseudohimantidium pacificum con Farrandula gibbula

Se encontró un total de 62 copépodos machos de la especie Farranula gibbula Giesbrecht, sin hallar hembras en las muestras. Esta especie es común en la Bahía de La Paz, particularmente durante la temporada de invierno (Palomares-García, 1996), cuando llega a alcanzar una talla de hasta $1 \mathrm{~mm}$ de longitud. F. gibbula ha sido registrado de diversas lagunas costeras de la costa occidental del Golfo de California y para el litoral oeste de la península de Baja California (Palomares-García et al., 1998).

De los 62 ejemplares de Farranula gibbula encontrados, 27 (43.5\%) presentaban sobre su caparazón a la diatomea Pseudohimantidium pacificum, perteneciente a la familia Protoraphidaceae, cuyos representantes son organismos señalados como típicamente epizoicos sobre distintas especies de copépodos (Gibson, 1979). Pseudohimantidium pacificum se puede confundir con otras especies y variedades descritas, pero se diferencia de ellas por presentar el eje apical curvado y un canal provisto de poros conspicuos en ambos extremos de la valva (González y Vergara, 1984); en este estudio se midieron 30 ejemplares de 28-70 $\mu \mathrm{m}$ de largo y de 10-17 $\mu \mathrm{m}$ de ancho, datos que se ajustan al intervalo de tallas descrito por Rivera et al. (1986).

Los valores promedio de abundancia de $P$. pacificum por muestra y por estación se resumen en el Cuadro 1; el menor número de epibiontes por copépodo se encontró en la estación 2 y el mayor en la 4; aunque el valor promedio más alto 
Cuadro 1. Número de individuos de copépodos con el epibionte Pseudohimantidium pacificum, porcentaje de infestación y por estación de muestreo.

\begin{tabular}{|l|l|l|l|l|l|l|}
\hline & Est. 1 & Est. 2 & Est. 3 & Est. 4 & Est. 5 & Est. 6 \\
\hline $\begin{array}{l}\text { Total de individuos de } F \text {. } \\
\text { gibbula por muestra }\end{array}$ & 8 & 2 & 12 & 20 & 8 & 12 \\
\hline $\begin{array}{l}\text { Cantidad y proporción de } \\
\text { individuos de F. gibbula } \\
\text { infestados por muestra }\end{array}$ & -- & $1(50 \%)$ & $4(33.3 \%)$ & $10(50 \%)$ & $4(50 \%)$ & $8(66.6 \%)$ \\
\hline $\begin{array}{l}\text { Promedio del número de } P \text {. } \\
\text { pacificum por copépodo }\end{array}$ & -- & 16 & 82 & 62.5 & 55 & 28 \\
\hline $\begin{array}{l}\text { Cantidad total de } P \text {. } \\
\text { pacificum por estación }\end{array}$ & -- & 16 & 328 & 625 & 220 & 224 \\
\hline
\end{tabular}

se registró en la 3 (82). Los individuos de $P$. pacificum se establecieron con mayor preferencia en el abdomen del copépodo (Figs. 2B, C y D), aunque también se observaron en el tórax y, en menor proporción, en la cabeza (Fig. 2E). La adhesión la realizan a través de un pedúnculo de mucopolisacáridos.

La infestación de diatomeas con copépodos reduce la movilidad de estos últimos, lo que ocasiona una disminución en su resistencia ante posibles depredadores e interfiere con su apareamiento (Hiromi et al., 1985). Por otro lado, Russell y Norris (1971) señalan que el hábitat de los copépodos puede ser responsable de que las diatomeas adheridas a los mismos pasen de un nivel trófico a otro y mencionan que la presencia de las últimas sobre el caparazón de los primeros puede incrementar su valor nutritivo. Por su parte, McClatchie et al. (1990) encontraron que algunos copépodos pueden llegar a tener grandes densidades de diatomeas adheridas, suficientes para registrar fluorescencia 25 veces mayor que la de los individuos no infestados; estos autores señalan que la presencia de diatomeas epizoicas interfiere en estudios sobre pastoreo, pues es posible que tengan implicaciones en la medición de tasas de alimentación a corto plazo, ya que éstas pueden ser sobreestimadas. Hargraves y Maranda (2002) registran colonias de P. pacificum cubriendo completamente los cuerpos de langostas juveniles en tanques de cultivo, provocando una alta mortandad de las mismas, por lo que se consideran como microalgas nocivas.

En las muestras colectadas de enero a noviembre de 2006 no se observó la presencia de copépodos infestados por la diatomea $P$. pacificum. El patrón de la temperatura del agua durante el año de 2006 mostró una oscilación de $12^{\circ} \mathrm{C}$, con el valor mínimo de $19^{\circ} \mathrm{C}$ en enero y el máximo de $31^{\circ} \mathrm{C}$ en agosto. La temperatura 

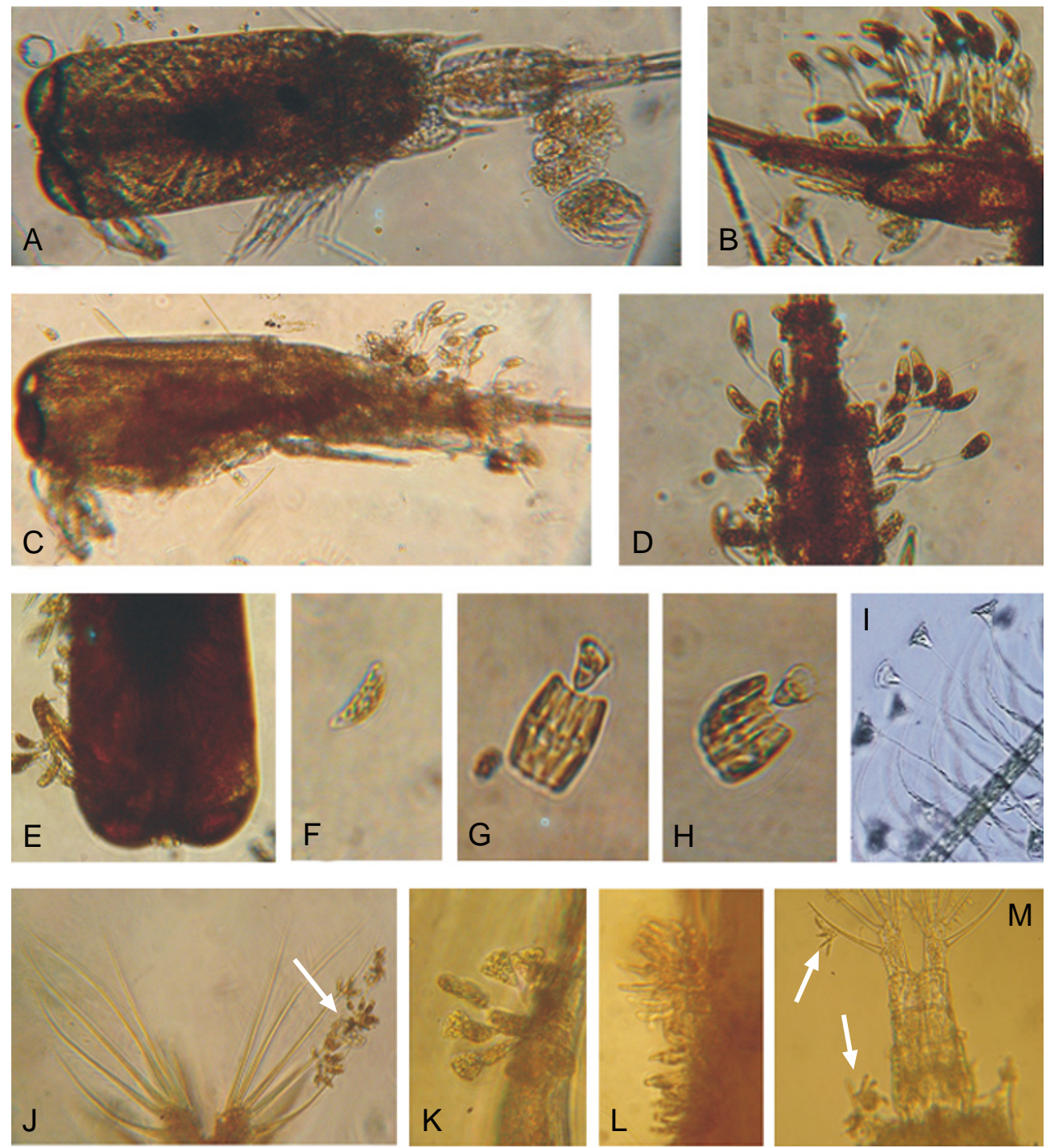

Fig. 2. (A) Ejemplar macho de Farranula gibbula que no presenta infestación de diatomeas. (B, C, D, E) Farranula gibbula mostrando la presencia de Pseudohimantidium pacificum en diversas partes del cuerpo. (F) célula aislada de Pseudohimantidium pacificum. (G, H) paquete de células de Fragilariopsis doliolus que presentan adherido un ciliado de tipo Vorticella. (I) relación simbiótica entre Chaetoceros coarctatus y Vorticella oceanica. (J) Diatomea penal (indicado con la flecha) sobre el copépodo Acartia claussii. (K) Licmophora sp. sobre un copépodo. (L, M) Diatomea penal (indicado con la flecha) sobre el copépodo Acartia lilljeborgii. 
registrada durante los días de muestreo, cuando se observó la presencia de la epibiosis diatomea/copépodo, fue de $24-25^{\circ} \mathrm{C}$, la cual es muy similar a la encontrada por Skovgaard y Saiz (2006) en Puerto Olímpico, España. En los muestreos realizados en los meses de enero de 2007 y febrero, septiembre y octubre de 2008 esta fue de 19, 20, 30 y $29^{\circ} \mathrm{C}$, respectivamente.

Pseudohimantidium pacificum se ha encontrado tanto en aguas templadas como tropicales de los océanos Pacífico, Índico y Atlántico, así como en el Mar Mediterráneo (Simonsen, 1974; Navarro, 1982; Skovgaard y Saiz, 2006). Hiromi et al. (1985) señalan que la distribución de la especie es mundial y puede ser considerada como de afinidad templado-tropical. La figura 3 muestra la distribución actual conocida de $P$. pacificum, la cual, a juzgar por el número de registros y su ubicación geográfica, tiene una mayor concentración en las aguas cálidas de ambas costas del Océano Pacífico. El registro de P. pacificum en este trabajo es el primero, tanto para la Bahía de La Paz, en el Golfo de California, como para el Pacífico Mexicano.

La epibiosis entre diatomeas y copépodos parece ser un hecho común en la Bahía de La Paz, ya que en muestras colectadas en junio de 2008 se observó un caso de epizoismo de la diatomea Licmophora sp. sobre un copépodo (Est. 4; Fig. 2K) (Gárate-Lizárraga et al., 2008).

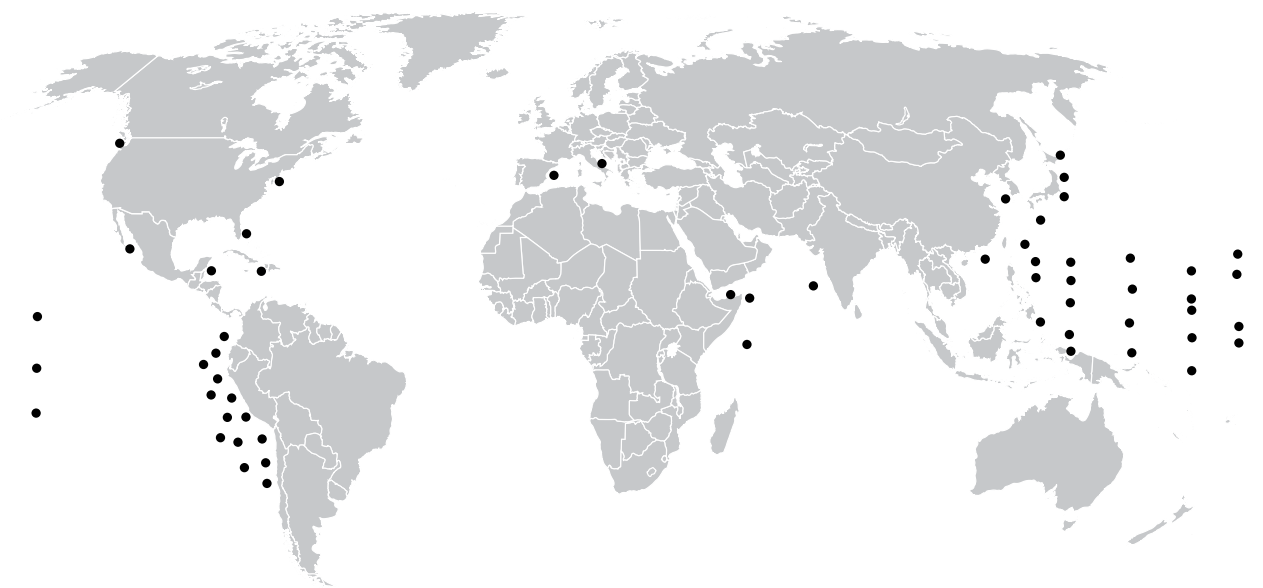

Fig 3. Distribución mundial de la diatomea epizoica Pseudohimantidium pacificum (•): Fuentes de información: Krasske, 1941; Russell y Norris, 1971; Belyaeva, 1973; Simonsen, 1970, 1974; Gibson, 1978, 1979; Navarro, 1982; Hiromi et al., 1985; Rivera et al., 1986; Lafforgue et al., 2001; Hanaoka et al., 2002; Hargraves y Maranda, 2002; Lee et al., 1993; Skovgaard y Saiz, 2006; Schiebel et al., 2004; este estudio. 
Otras asociaciones

En las muestras de febrero de 2008 se observó la presencia de una diatomea penal distribuida en forma individual o formando ramilletes de entre $10 \mathrm{y} \approx 60$ células en diversas partes del cuerpo de tres ejemplares de copépodos de la especie Acartia clausii Giesbrecht (Est. 1 y 3; Fig. 2J), mientras que en septiembre de este mismo año se le registró en seis individuos de Acartia lilljeborgii Giesbrecht (Est. 2 y 3; Fig. 2L, M), ambas especies comunes en la Bahía de la Paz. Estos hallazgos, también representan el primer registro de diatomeas epizoicas sobre especies del género Acartia en aguas mexicanas.

En las muestras analizadas se encontraron asimismo las siguientes asociaciones: la diatomea Chaetoceros coarctatum Lauder con el ciliado Vorticella oceanica Zacharias (Fig. 2I); Leptocylindrus mediterraneus (H. Peragallo) Hasle y el flagelado Solenicola setigera Pavillard; y la asociación entre Fragilariopsis doliolus (Wallich) Medlin \& Sims y un protozoario del tipo Vorticella (Figs. 2G, H), así como una relación similar entre el silicoflagelado Dictyocha pentagona (Schulz) Bukry \& Foster y el mismo ciliado. De estas dos últimas asociaciones no se tenían registros en la literatura mundial, por lo que resultan hallazgos importantes.

Otros casos de epifitismo, endosimbiosis y parasitismo

En un estudio previo realizado por Garate-Lizárraga y Martínez López (2004) en la Bahía de La Paz se registraron 17 casos de asociación epifítica entre coanoflagelados y diatomeas, siendo los más frecuentes los relacionados con la diatomea del género Chaetoceros, al que le siguen Rhizosolenia, Guinardia, Bacteriastrum, Coscinodiscus, Pseudosolenia y Corethron. En nuestra investigación hemos encontrado varios casos de epifitismo de diatomeas penales sobre dos especies de Chaetoceros, y de una de cocolitofórido sobre entidades de los géneros Chaetoceros y Bacteriastrum.

En las diatomeas Hemiaulus sinensis Greville, H. hauckii Grunow in Van Heurck, H. membranaceus Cleve, Rhizosolenia imbricata Brightwell, $R$. cleveii var. communis Sundström, Guinardia cylindrus (Cleve) Hasle, Pseudosolenia calcar-avis (Schultze) Sundström y Chaetoceros compressus Lauder se presentó endosimbiosis con la cianobacteria Richelia intracellularis Schmidt.

Asimismo, se han registrado casos de parasitismo del dinoflagelado Paulsenella chaetoceratis (Paulsen) Chatton sobre especies del género Chaetoceros y del dinoflagelado Amoebophrya ceratii (Koeppen) Cachon infestando a Gonyaulax polygramma Stein (Gárate-Lizárraga y Martínez-López, 2004; Gárate-Lizárraga et al., 2006). 
Por su parte, cianobacterias cocoides fueron observadas en epibiosis con Ornithocercus magnificus Stein. De la última especie y de O. heteroporus Kofoid y $O$. steinii Schütt se tienen registros de este tipo de asociación en el Sistema Lagunar Magdalena-Almejas (Gárate-Lizárraga et al., 2007).
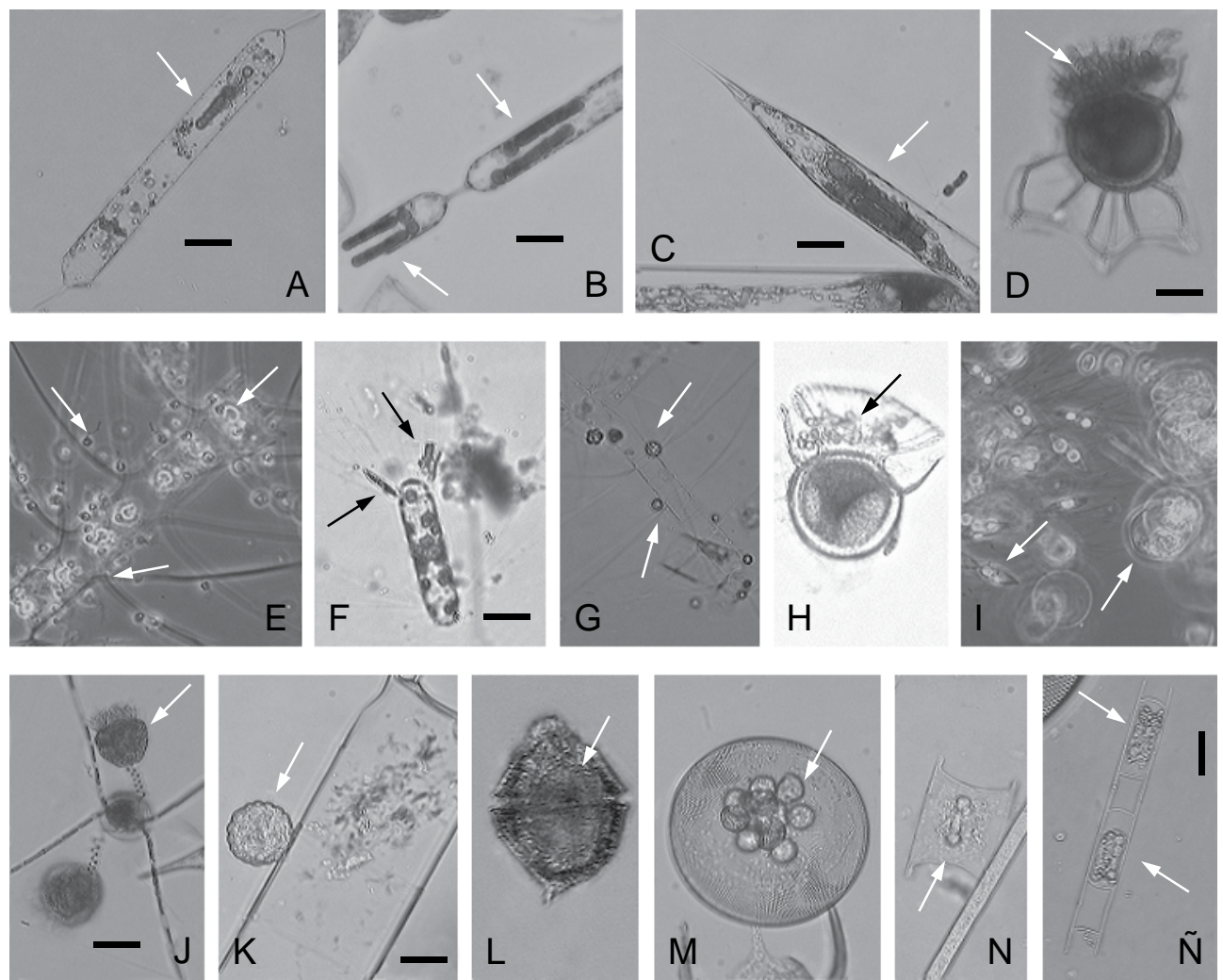

Fig 4. Diferentes tipos de simbiosis observadas entre microalgas y protozoarios en la Bahía de La Paz. Asociación de Guinardía cylindrus (A y B), Rhizosolenia clevei var. communis Sundström (C), Hemiaulus sinensis (N), Hemiaulus hauckii (Ñ) con Richelia intracellularis (indicada con la flecha). Ornithocercus magnificus mostrando la presencia de cianobacterias (D y H). Coanoflagelados cubriendo la superficie valvar de Chaetoceros didymus (E). Corethron hystrix Hensen con diatomeas epifitas (F). Chaetoceros sp. con cocolitofóridos epifitos (G). Epibiosis entre Chaetoceros sp. y Thalassiosira sp. con diatomeas epifitas del género Pseudo-nitzschia (I). Chaetoceros coarctatum (J), Biddulphia sp. (K) y Coscinodiscus asteromphalus Ehrenberg (M) con Vorticella oceanica. Gonyaulax polygramma parasitado por Amoebophrya ceratii (L, indicado con la flecha). Barra de escala $=20 \mu \mathrm{m}$. 
Recientemente, durante los florecimientos de las poblaciones de Chaetoceros socialis Lauder ocurridos en febrero de $2007\left(\approx 3,600000\right.$ cél L $\left.^{-1}\right)$ y enero y febrero del $2008\left(\approx 800,000\right.$ - 4, 200000 cél $\left.\mathrm{L}^{-1}\right)$ se observó la presencia de diatomeas penales epifitas pertenecientes al género Pseudo-nitzschia sobre las valvas de $C$. socialis. Este ejemplo de epifitismo también lo han registrado Alonso-Rodríguez et al. (2008) para diversas lagunas costeras de Sinaloa, y la asociación ha sido documentada para las costas de las Islas San Juan, Washington por Rines et al. (2002).

En la figura 4 se ilustran algunos de los casos más representativos de epibiosis, endosimbiosis y parasitismo entre diversas especies de microalgas y ciliados que se encuentran en la Bahía de la Paz.

\section{CONCLUSIONES}

Los resultados de este estudio permiten concluir que las asociaciones simbióticas entre las diferentes especies de microalgas con cianobacterias, ciliados y copépodos son frecuentes en aguas del Golfo de California, particularmente en la Bahía de La Paz. Tales asociaciones no sólo están conformadas por diatomeas y dinoflagelados que se adhieren a otros organismos del fitoplancton o del zooplancton, sino también de ciliados y cianobacterias. La endosimbiosis y el parasitismo son tipos de simbiosis que no están muy estudiados en nuestras áreas de estudio, por lo cual representan una línea de investigación con un gran potencial. En futuras investigaciones es necesario considerar los diferentes tipos de asociaciones simbióticas que se establecen entre las microalgas planctónicas y otros grupos del zooplancton a fin de tener un mayor conocimiento y sobre el tipo y la dinámica de las mismas en el plancton marino del Pacífico Mexicano.

\section{AGRADECIMIENTOS}

Se agradece al Instituto Politécnico Nacional por el apoyo financiero para la realización de este trabajo a través de los proyectos SIP-20071252, SIP-20070883 y SIP-20082279; a Ricardo Palomares-García y Gabriela M. Esqueda-Escárcega (Centro Interdisciplinario de Ciencias Marinas, del Instituto Politécnico Nacional) por su ayuda en la identificación de las especies de copépodos, a María Clara Ramírez-Jáuregui (Instituto de Ciencias del Mar y Limnología, Unidad Mazatlán, de la Universidad Nacional Autónoma de México) por facilitar la literatura especializada 
y a A. Martínez (también del Centro Interdisciplinario de Ciencias Marinas, del Instituto Politécnico Nacional ) por su ayuda en la toma de las fotografías que aparecen en la Figura 4. Asimismo, agradecemos la meticulosa revisión editorial y de dos árbitros anónimos a nuestro manuscrito. El primer autor es becario de la Comisión de Cooperación y Fomento de Actividades Académicas y de los Estímulos al Desempeño de los Investigadores.

\section{LITERATURA CITADA}

Alonso-Rodríguez, R., I. Gárate-Lizárraga y S. Gómez-Aguirre. 2003. Epifitismo del ciliado Vorticella sp. (Ciliophora: Peritricha) sobre tres especies de diatomeas, en la Bahía de Mazatlán, México. X Congreso Latinoamericano de Ciencias del Mar. San José, Costa Rica. Disco compacto.

Alonso-Rodríguez, R., D. Hernández-Becerril e I. Gárate-Lizárraga. 2008. Catálogo de microalgas de las lagunas costeras de Sinaloa. In: Páez-Osuna, F. (ed.). Lagunas Costeras de Sinaloa. Universidad Nacional Autónoma de México, Instituto de Ciencias del Mar y Limnología (Estación Mazatlán), Colegio de Sinaloa, Comité Estatal de Sanidad Acuícola de Sinaloa, Secretaría de Medio Ambiente y Recursos Naturales, y Consejo Nacional de Ciencia y Tecnología. Culiacán. 198 pp.

Belyaeva, T. V. 1973. Distribution of the diatoms of the genus Pseudohimantidium Hustedt et Krasske. Bot. Zh. (Leningrad) 58(6): 883-885.

Carman, K. R. y F. C. Dobbs. 1997. Epibiotic microorganisms on copepods and other marine crustaceans. Microsc. Res. Technol. 37: 116-135.

Cruz-Orozco, R., A. Mendoza-Maravillas y C. Martínez-Noriega. 1989. Profundidades y formas de la Bahía de La Paz. Geonotas 1: 2.

Coats, D. W., T. Bachvaroff, S. M. Handy, S. Kim, I. Gárate-Lizárraga y C. F. Delwiche. 2008. Prevalence and phylogeny of parasitic dinoflagellates (genus Blastodinium) infecting copepods in the Gulf of California. Oceánides 23(1-2): 67-77.

Cupp, E. E. 1943. Marine plankton diatoms of the west coast of North America. Bull. Scripps Inst. Oceanogr. 5: 1-238.

De Silva-Dávila, R. y R. Palomares-García. 2002. Distributional patterns of the euphausiid community in Bahía de La Paz, B.C.S., México. In: Hendrickx, M. E. (ed.). Contributions to the study of the East Pacific crustaceans. Instituto de Ciencias del Mar y Limnología, Universidad Nacional Autónoma de México. México, D.F. pp. 109-125.

Gárate-Lizárraga, I. y D. A. Siqueiros-Beltrones. 1998. Time variations in phytoplankton assemblages in a subtropical lagoon system after the 1982/83 El Niño event (1984/86). Pac. Sci. 52: 79-97.

Gárate-Lizárraga, I. y D. A. Siqueiros-Beltrones. 2003. Infection of Ceratium furca by the parasitic dinoflagellate Amoebophrya ceratii (Amoebophridae) in the Mexican Pacific. Acta Bot. Mex. 65: 1-9. 
Gárate-Lizárraga, I., D. A. Siqueiros-Beltrones y C. A. Lechuga-Devéze. 1990. Estructura de las asociaciones microfitoplanctónicas de la región central del Golfo de California en el otoño de 1986. Cienc. Mar. 16(3): 131-153.

Gárate-Lizárraga, I. y A. Martínez-López. 2004. Simbiosis en diatomeas y dinoflagelados de la porción sur del Golfo de California. XIII Reunión Nacional de la Sociedad Mexicana de Planctología y VI Reunión Internacional de Planctología. Nuevo Vallarta, Nayarit, México. pp. 95-96.

Gárate-Lizárraga, I., M. S. Muñetón-Gómez y V. Maldonado-López. 2006. Florecimiento del dinoflagelado Gonyaulax polygramma frente a la Isla Espíritu Santo, Golfo de California (Octubre-2004). Rev. Invest. Mar. 27(1): 31-39.

Gárate-Lizárraga, I., C. J. Band-Schmidt, G. Verdugo-Díaz, M. S. Muñetón-Gómez y E. F. Félix-Pico. 2007. Dinoflagelados (Dinophyceae) del sistema lagunar MagdalenaAlmejas. In: Funes-Rodríguez, R., J. Gómez-Gutiérrez y R. Palomares-García (eds.). Estudios ecológicos en Bahía Magdalena. Centro Interdisciplinario de Ciencias Marinas - Instituto Politécnico Nacional. La Paz. pp. 145-174.

Gárate-Lizárraga, I., C. J. Band-Schmidt y T. Grayeb del Alamo. 2008. Myrionecta, Gyrodinium and Katodinium bloom in Gulf of California. Harmful Algae News, An IOC Newsletter on toxic algae and algal blooms. Paris. 37: 6-7.

Gibson, R. A. 1978. Pseudohimantidium pacificum, an epizoic diatom new to Florida Current (western North Atlantic Ocean.). J. Phycol. 14: 371-373.

Gibson, R. A. 1979. An ultrastructure study of Pseudohimantidium pacificum Hust. \& Krasske (Bacillariophyceae: Photoraphidaceae) with special reference to the labiate processes. Nova Hedwigia Beih. 64: 147-156.

González, H. y L. Vergara. 1984. Interacción ecológica entre la diatomea epizoica Pseudohimantidium pacificum Hust. \& Krasske 1941 y copépodos del género Corycaeus. Rev. Biol. Mar. 20(1): 77-90.

Green, J. 1974. Parasites and epibionts of Cladocera. Trans. Zool. Sot. Lond. 32: 417-515.

Hanaoka, H., S. Ohtani y S. Ohtsuka. 2002. A study of life history of pennate diatom Pseudohimantidium pacificum attached to planktonic copepod Corycaeus affinis. Diario de la Reunión Anual. Japan Soc. for Science Education 2(6): 263-264.

Hargraves, P. E. y L. Maranda. 2002. Potentially toxic or harmful microalgae from the northeast coast. Northeast. Nat. 9(1): 81-120.

Hiromi, J., S. Kadota y H. Takano. 1985. Diatom infestation of marine copepods (Review). Bull. Tokai Reg. Fish. Res. Lab. 117: 37-45.

Ho, J. y P. S. Perkins. 1985. Symbionts of marine copepoda: an overview. Bull. Mar. Sci. 37: 586-598.

Krasske, G. 1941. Die Kieselalgen des chilenischen Küstenplanktons. Arch. Hydrobiol. 38: 260-287.

Lafforgue, M., C. Pateron, V. Salvert y M. Ricard. 2001. Proyecto manejo ambiental de las Islas de La Bahía. Informe técnico No. Cac 03 - Volumen 2/2. Diagnóstico de la calidad de las aguas de la Isla de Guanaja, Honduras. Isla de Guanaja, Honduras. 98 pp.

Lee, J. H., J. Y. Lee y M. O. Kim. 1993. The fine structure of the marine epizoic pennate diatom Pseudohimantidium pacificum in Korean coastal waters. Jour. Oceanol. Soc. Kor. 28(3): 202-211. 
Lincoln, R. J., G. A. Boxball y P. F. Clark. 1995. Diccionario de ecología, evolución y taxonomía. Fondo de Cultura Económica. México, D.F. 488 pp.

McClatchie, S., R. Kawachi y D. E. Dalley. 1990. Epizoic diatoms on the euphausiid Nyctiphanes australis: consequences for gut pigment analyses of whole krill. Mar. Biol. 104: 227-232.

Navarro, J. N. 1982. A survey of the marine diatoms of Puerto Rico. IV. Suborder Araphidineae: families Diatomaceae and Protoraphidaceae. Bot. Mar. 25: 247263.

Ohtsuka, S., M., Hora, T. Suzaki, M. Arikawa, G. Omura y K. Yamada. 2004. Morphology and host-specificity of the apostome ciliate Vampyrophrya pelagica infecting pelagic copepods in the Seto Inland Sea, Japan. Mar. Ecol. Prog. Ser. 282: 129142.

Palomares-García, R. 1996. Estructura espacial y variación estacional de los copépodos en la Ensenada de La Paz. Oceánides 11(1): 29-43.

Palomares-García, R., E. Suárez y S. Hernández-Trujillo. 1998. Catálogo de los copépodos (Crustacea) pelágicos del Pacífico mexicano. Centro Interdisciplinario de Ciencias Marinas - El Colegio de la Frontera Sur. México, D.F. 52 pp.

Rines, J. E. B., P. L. Donaghay, M. M. Dekshenieks, J. M. Sullivan y M. S. Twardowski. 2002. Thin layers and camouflage: hidden Pseudo-nitzschia populations in a fjord in the San Juan Islands, Washington, USA. Mar. Ecol. Prog. Ser. 225: 123-137.

Rivera, P. S., H. E. González y H. L. Barrales. 1986. Cingulum and valve morphology of Pseudohimantidium Hustedt \& Krasske (Bacillariophyceae). Phycologia 25: 1927.

Russell, D. J. y R. E. Norris. 1971. Ecology and taxonomy of an epizooic diatom. Pac. Sci. 25: 357-367.

Schiebel, R., A. Zeltner, U. F. Treppke, J. J. Waniek, J. Bollmann, T. Rixen y C. Hemleben. 2004. Distribution of diatoms, coccolithophores and planktic foraminifers along a trophic gradient during SW monsoon in the Arabian Sea. Mar. Micropaleon. 51(3-4): 345-371.

Simonsen, R. 1970. Protoraphidaceae, eine neue Familie der Diatomeen. Nova Hedwigia Beih. 31: 377-394.

Simonsen, R. 1974. The diatom plankton of the Indian Ocean Expedition of the R.V. "Meteor. 1964-65”. Meteor. Forschungsergeb. (D. Biol.). 19: 1- 66.

Siqueiros-Beltrones, D. A., S. Ibarra-Obando y D. Loya-Salinas. 1985. Una aproximación a la estructura florística de las diatomeas epifitas de Zostera marina y sus variaciones temporales en Bahía Falsa, San Quintín, B.C. Cien. Mar. 11(3): 69-88.

Siqueiros-Beltrones, D. A., E. Serviere-Zaragoza y U. Argumedo-Hernández. 2002. Epiphytic diatoms of Macrocystis pyrifera (L.) C. Agardh from the Baja California Peninsula, Mexico. Oceánides 17(1): 31-39.

Siqueiros-Beltrones, D. U. y U. Argumedo-Hernández. 2005. Florística de diatomeas epifitas en laminales apicales de Macrocystis pyrifera (L.) C. Agardh. Oceánides 20(1,2): 3763.

Skovgaard, A. y E. Saiz. 2006. Seasonal occurrence and role of protistan parasites in coastal marine zooplankton. Mar. Ecol. Prog. Ser. 327: 37-492. 
Takano, H. 1983. New and rare diatoms from Japanese marine waters. XI. Three new species epizoic on copepods. Bull. Tokai Reg. Fish. Res. Lab. 111: 23-33.

Utermöhl, H. 1958. Zur Vervollkommnung der quantitativen Phytoplankton-Methodik. Mitt. Int. Verein Theor. Angew. Limnol. 5: 567-596.

Villaseñor-Casales, A. 1979. Distribución vertical de temperatura, salinidad y oxígeno disuelto en la Bahía de La Paz, Baja California Sur, durante la primavera de 1976. Calif. Coop. Ocean. Fish. Invest. Rep. 20: 146-149. 
